\title{
ORIGINAL RESEARCH \\ Asymmetric Mineralization of the Arytenoid Cartilages in Patients without Laryngeal Cancer
}

E. Zan

D.M. Yousem

N. Aygun
BACKGROUND AND PURPOSE: Sclerosis of the arytenoid cartilage may be seen as an incidental finding in patients who do not have laryngeal cancer but may also be an early sign of neoplastic infiltration. Our purpose was to determine the frequency of asymmetric mineralization, in particular sclerosis, of the arytenoid cartilages on CT scans in adults who have no history of laryngeal cancer.

MATERIALS AND METHODS: Cervical CT scans of 972 consecutive patients seen in our emergency department were retrospectively evaluated. Three hundred twenty-two patients were excluded who were younger than 18 years of age or whose arytenoids could not be reliably seen due to artifacts. Six hundred fifty patients (424 men, 226 women) were assessed, and their arytenoid cartilages were graded as nonmineralized, calcified, sclerotic, or ossified on each side separately. The mean age of patients was $44.3 \pm 17.8$ years (range, $18-97$ years).

RESULTS: The frequencies of asymmetric arytenoid cartilage sclerosis, calcification, and ossification were $4.9 \%$ (32/650), 4.4\% (29/650), and 3.4\% (23/650), respectively, with an overall asymmetric mineralization frequency of $12.9 \%(84 / 650)$. Asymmetric sclerosis was more common in women $(16 / 226,7.1 \%)$ than in men $(16 / 424,3.8 \%)$, but the difference was just at statistical significance $(P=$ .05). The rate of unilateral arytenoid sclerosis was $4.6 \%$ in all subjects, $6.6 \%$ in women, and $3.5 \%$ in men. Unilateral sclerosis is much more frequently associated with the left arytenoid than the right.

CONCLUSIONS: Asymmetric mineralization of the arytenoid cartilages was seen in $12.9 \%$ of our study population. This should be taken into account when evaluating CT scans of patients with laryngeal cancer for arytenoid cartilage invasion to avoid false-positive reads.

ABBREVIATIONS: CAJ $=$ cricoarytenoid joint

$\mathbf{A}^{\mathrm{r}}$ rytenoid cartilage invasion by squamous cell carcinoma of the larynx has important bearings on treatment. ${ }^{1-3}$ While arytenoid mobility on clinical examination remains the primary parameter for the determination of whether the arytenoid will be included in a laryngectomy specimen, it is not always easy to ascertain the status of the arytenoid cartilage on physical/endoscopic examination alone. The CT findings suggestive of arytenoid cartilage invasion include erosive, lytic, and sclerotic changes as well as soft-tissue tumor abutting or engulfing the cartilages. ${ }^{2}$ Sclerosis of the arytenoids may be seen as an incidental finding, however, in patients who do not have laryngeal cancer. ${ }^{4}$ When evaluating a patient with laryngeal cancer and unilateral arytenoid sclerosis, it is difficult to determine whether sclerosis is due to tumor invasion, reactive change, or normal variation. Taken from the opposite standpoint, asymmetric lack of calcification may be interpreted as a lytic process.

Our purpose was to determine the frequency of asymmetric mineralization, in particular sclerosis, of the arytenoid cartilages on CT scans in adults who have no history of laryngeal cancer.

Received July 19, 2010; accepted after revision October 16 .

From the Department of Radiology (E.Z.), Ataturk Education and Research Hospital, Ankara, Turkey; and Department of Radiology (D.M.Y., N.A.), Johns Hopkins Medical Institutions, Baltimore, Maryland.

Paper previously presented at: Annual Meeting of the American Society of Neuroradiology, May 16-21, 2009; Vancouver, British Columbia, Canada.

Please address correspondence to Nafi Aygun, MD, Johns Hopkins Medical Institutions, 600 N. Wolfe St, Phipps B 112B, Baltimore, MD 21287-2182; e-mail: naygun1@jhmi.edu DOI 10.3174/ajnr.A2444

\section{Materials and Methods}

We retrospectively evaluated 972 consecutive cervical spine CT scans of patients seen in our emergency department from August to October 2008. The most common indications for these CT scans were trauma and neck pain. Three hundred twenty-two patients were excluded who were younger than 18 years of age or whose arytenoids could not be reliably seen due to artifacts. A total of 650 patients ( 424 men, 226 women) were assessed; the mean age was $44.3 \pm 17.8$ years (range, 18-97 years). Our institutional review board approved the retrospective review of patient data for this study. Informed consent was waived by the institutional review board, and the study was compliant with the Health Insurance Portability and Accountability Act.

CT scans were obtained on a 64-section scanner (Siemens, Erlangen, Germany) at 1-mm intervals with images reconstructed by using both bone and soft-tissue algorithms. Interpretation was made on 1-mm axial images, and multiplanar reformats were performed to better visualize the arytenoids as deemed necessary by the interpreter, 1 of the investigators (E.Z., fourth-year radiology resident).

Mineralization of the arytenoid cartilages was categorized as nonmineralization, calcification, sclerosis, and ossification (Fig 1). When the arytenoid cartilage demonstrated soft-tissue attenuation, it was determined to be "nonmineralized." "Calcification" was defined as high attenuation observed within the cartilage (Fig 1A), which was not as bright as sclerosis. "Sclerosis" was defined as a prominent chalklike attenuated appearance of any part of the cartilage, which may or may not obliterate the marrow space in the body of the arytenoid cartilage (Fig $1 B-D$ ). "Ossification" was defined as a high-attenuation periphery of the cartilage with low-attenuation central bone marrow (Fig 1C). Separation of calcification from sclerosis was based on subjective evaluation. No Hounsfield unit measurement was obtained because this would be hampered by partial volume averaging. When there was ambiguity, 2 observers (E.Z., a 

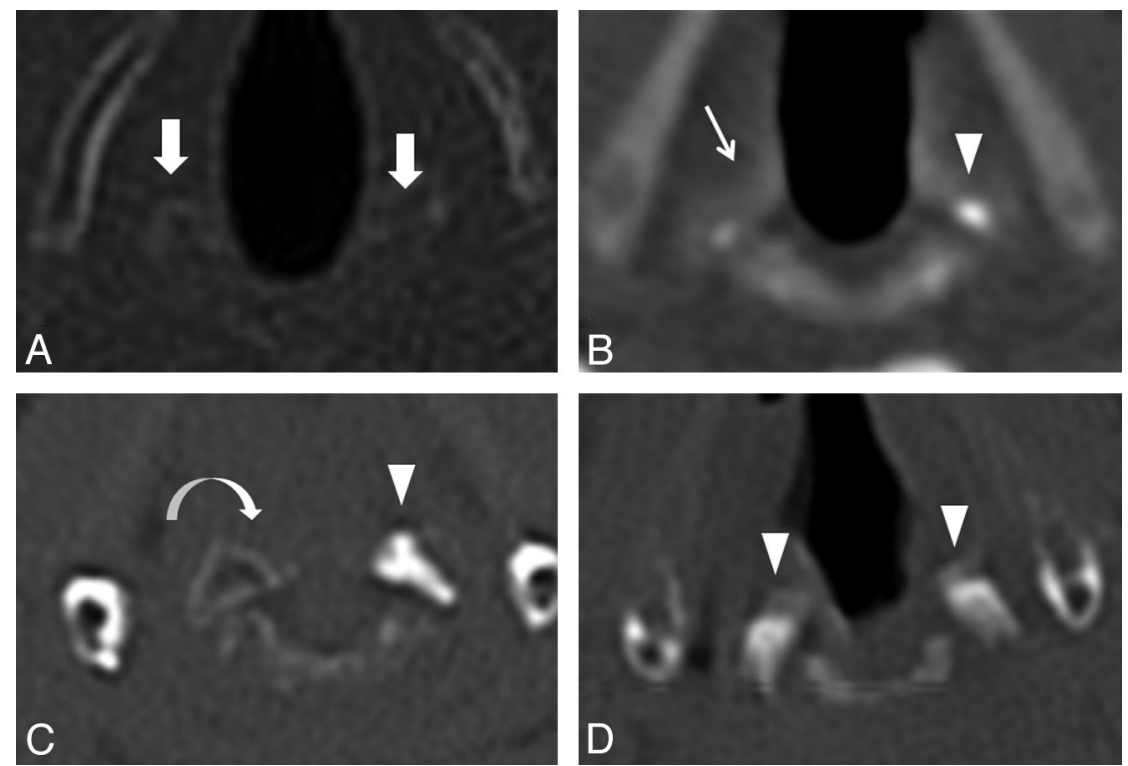

Fig 1. Axial CT scans of 4 different patients at the level of arytenoid cartilages. A, Bilateral nonmineralized arytenoid cartilages (block arrows). B, Calcified right (arrow) and sclerotic left (arrowhead) arytenoid cartilages. C, Ossified right (curved arrow) and sclerotic left (arrowhead) arytenoid cartilages. D, Bilateral sclerotic arytenoid cartilages (arrowheads).

\begin{tabular}{lcc}
\hline \multicolumn{3}{l}{ The rate of different types of mineralization } \\
\hline $\begin{array}{l}\text { Type of } \\
\text { Mineralization }\end{array}$ & $\begin{array}{c}\text { No. of } \\
\text { Mineralizations }\end{array}$ & $\begin{array}{c}\text { No. of } \\
\text { Asymmetric } \\
\text { Mineralizations }\end{array}$ \\
\hline Ossification & $275(42.3 \%)$ & $29(4.5 \%)$ \\
Sclerosis & $126(19.4 \%)$ & $32(4.9 \%)$ \\
Calcification & $145(22.3 \%)$ & $23(3.5 \%)$ \\
No mineralization at all & $104(16 \%)$ & $\mathrm{N} / \mathrm{A}$ \\
Total & 650 & $84(12.9 \%)$ \\
\hline
\end{tabular}

fourth-year radiology resident, and N.A., a certified neuroradiologist with 10 years of experience) reviewed the case and reached a consensus.

The term "asymmetric sclerosis" of an arytenoid pair was used when 1 arytenoid cartilage was classified as sclerotic and the other was classified as either nonmineralized, calcified, or ossified. If there was bilateral sclerosis occurring in different portions of the arytenoids, it too was labeled "asymmetric sclerosis."

The term "asymmetric calcification" was used in the same fashion to identify cases in which there was calcification on 1 side but not the other or when the calcification was asymmetric from side to side. Cases of asymmetric sclerosis were excluded from consideration in the asymmetric calcification analysis. We graded cases of asymmetric ossification in the same way, looking for the presence or absence or asymmetric locations of ossification, excluding sclerotic cases.

Finally, the term "asymmetric mineralization" was used as a broader all-inclusive categorization when any asymmetry was present. Thus, this term was used to indicate combinations of calcification sclerosis or ossification that were present on 1 side but not the other or present at different locations in the arytenoid cartilages.

The right and left arytenoid cartilages were scored separately. Findings were evaluated on the basis of patients' sex and age groups for each decade.

All of the scans were evaluated by 1 of the investigators familiar with cross-sectional studies of the head and neck. When there were areas of ambiguity, a second interpreter was used to obtain a consen-

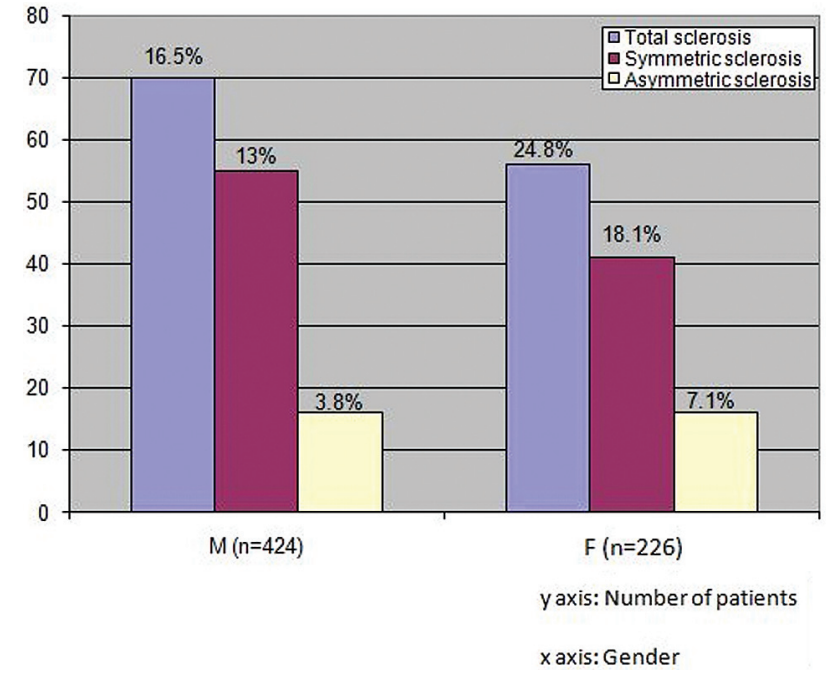

Fig 2. Sex-specific rates of total arytenoid sclerosis compared with symmetric and asymmetric sclerosis.

sus reading. This second reviewer also reviewed all 84 instances of asymmetric mineralization.

\section{Results}

The overall frequency of asymmetric arytenoid cartilage mineralization of any kind was $12.9 \%$ (84/650) (Table). When comparison was based on sex, the overall asymmetry was $13.2 \%(30 / 226)$ in female and $12.7 \%(54 / 424)$ in male patients.

\section{Sclerosis}

The overall rate of arytenoid sclerosis was 19.4\% (126/650) in all subjects. The overall rate of sclerosis was statistically significantly higher $(P=.008)$ in women $(56 / 226,24.8 \%)$ than in men $(70 / 424,16.5 \%)$ (Fig 2). Asymmetric sclerosis (32/650 overall, $4.9 \%$ ) was more common in women (16/226, 7.1\%) when compared with men $(16 / 424,3.8 \%)$, but the difference 


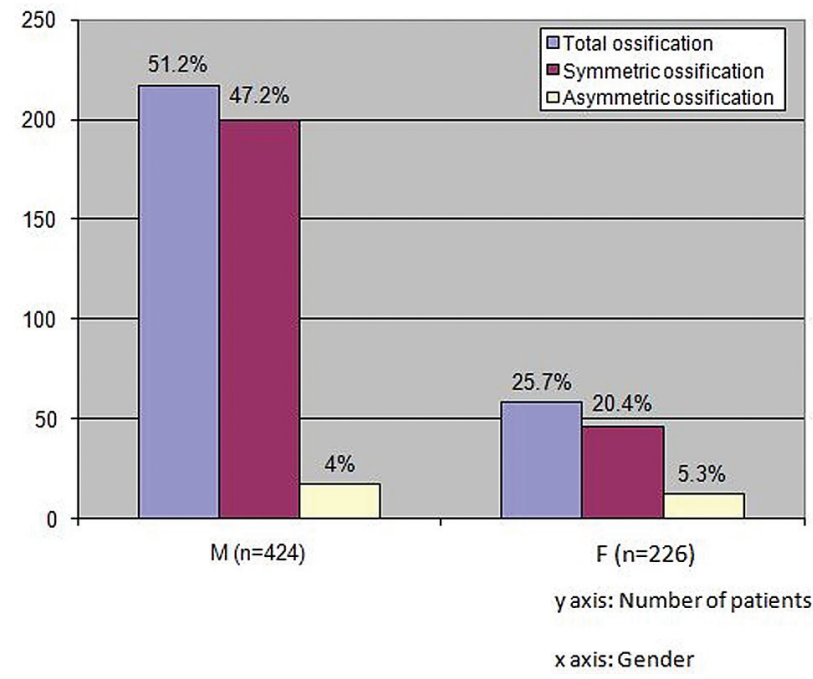

Fig 3. Sex-specific rates of total arytenoid ossification compared with symmetric and asymmetric ossification.

was just above statistical significance $(P=.05)$. In all cases of asymmetric sclerosis, the nonsclerotic arytenoid showed 1 form of mineralization, and there was no case of sclerosis on 1 side and nonmineralized arytenoid on the other. The lateralization of asymmetry was to the right in 5 patients ( 1 man, 4 women; $16.6 \%$ of all unilateral sclerotic arytenoids) and to the left in 25 patients (13 men, 12 women; $83.3 \%$ of all the unilateral sclerosis). Within the asymmetric sclerosis group, only 2 had bilateral but asymmetric sclerosis that was not dominant on either side. The prevalence of symmetric sclerosis had no statistically significant difference between sexes $(P=.05)$.

\section{Ossification}

Ossification was evident in 275 (42.3\%) patients. Ossification was dominantly observed in 217 men (51.2\%) over 58 women $(25.7 \%)$, and the difference between sexes was statistically significant $(P=.00)$ (Fig 3$)$. The asymmetric ossification rate did not statistically significantly differ between sexes $(5.3 \%, n=$ 12 in women versus $4 \%, n=17$ in men, $29 / 650 ; P=.28)$. Symmetric ossification was dominant in men $(47.2 \%, n=200$ versus $20.4 \%, n=46$ in women, $P=.00$ ).

\section{Calcification}

Calcification was evident in 145 (22.3\%) patients, and women showed statistically higher calcification rates $(27.4 \%, n=62$, versus $19.6 \%, n=83 ; P=.00$ ) (Fig 4 ). The rate of asymmetric calcification was $3.4 \%$ (23/650). There was no statistically significant difference between sexes for asymmetric calcification (female, $4.4 \%, n=10$ versus male, $3 \%, n=13$ ).

\section{Age Groups}

When evaluations were made on the basis of age groups for sclerosis, it was observed that the overall rate of sclerosis increased with age, with a peak demonstrated in the fourth decade (Fig 5). The difference between age groups by decades was statistically significant $(P=.00)$. The increased rates with age were present whether we were evaluating asymmetric or symmetric sclerosis $(P=.00$, each). Evaluation of the rate of

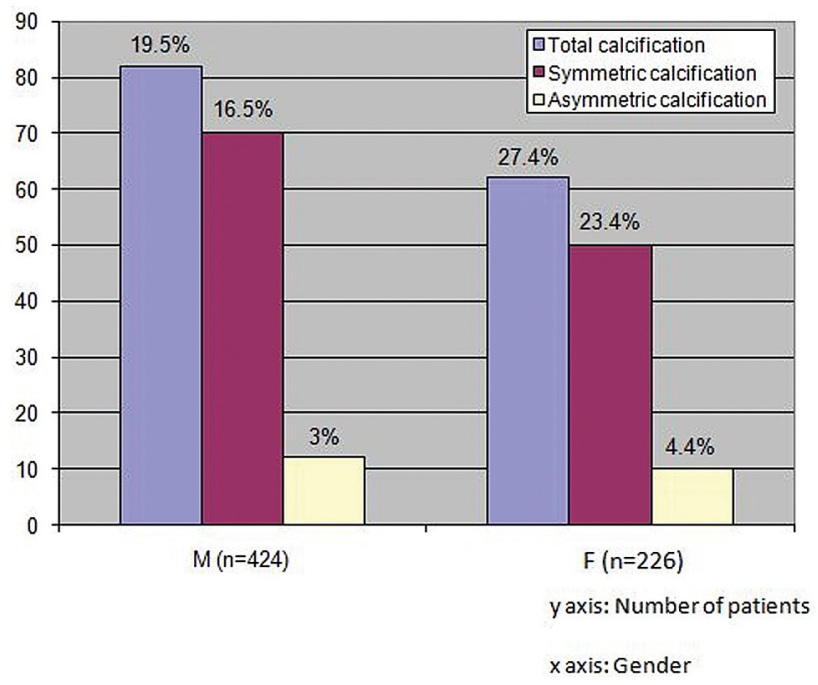

Fig 4. Sex-specific rates of total arytenoid calcification compared with symmetric and asymmetric calcification.

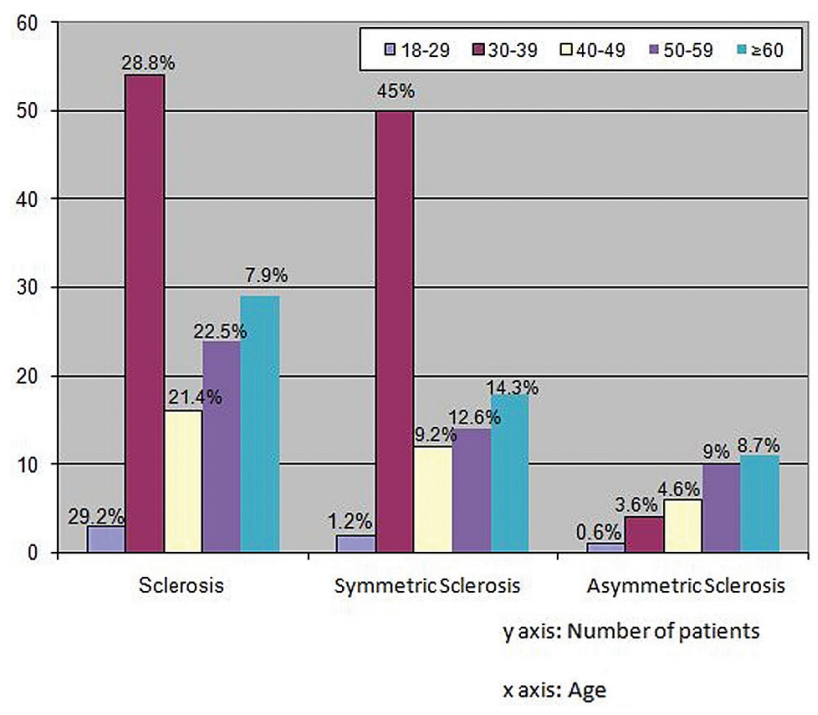

Fig 5. Age-specific rates of arytenoid sclerosis

ossification and age groups showed that the overall rate of ossification increased with age, and the difference between age groups was statistically significant $(P=.00)$, with the exception of the fourth decade, which showed a decreased rate of ossification compared with the third decade (Fig 6).

The rate of arytenoid cartilage calcification decreased with age with a more significant drop seen at the seventh decade (Fig 7). The decrease was statistically significant both for symmetric and asymmetric calcification $(P=.00)$.

There was no statistically significant difference in overall asymmetric mineralization of the cartilages for different age groups $(P=.49)$ (Fig 8$)$.

\section{Sex}

The difference for each type of mineralization in each age group based on sex was also evaluated. Sclerosis was statistically significantly higher $(P=.01)$ in women for all ages except the fourth decade, when it was more common in men by a statistically significantly higher rate $(P=.00)$. When we 


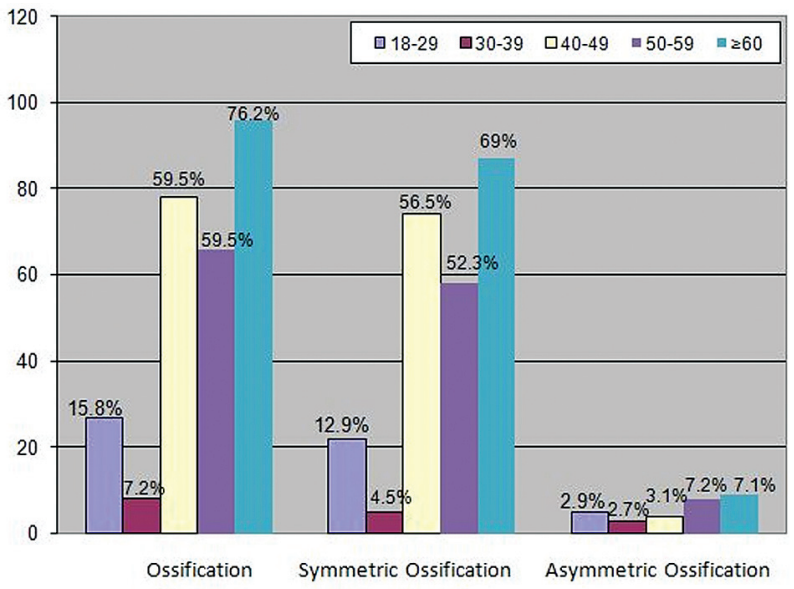

y axis: Number of patients

$x$ axis: Age

Fig 6. Age-specific rates of arytenoid ossification.

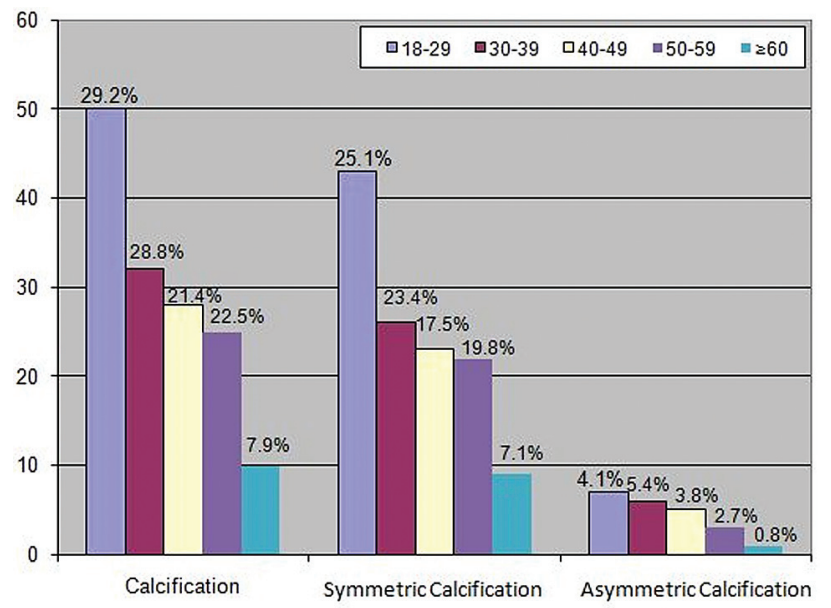

y axis: Number of patients

$x$ axis: Age

Fig 7. Age-specific rates of arytenoid calcification

looked at the asymmetric sclerosis rates, the only significant difference was observed in the patients older than 60 years of age, favoring the men $(P=.03)$

The rate of ossification was statistically significantly higher $(P=.02)$ in men for all decades of life except the fourth decade, for which the difference was not statistically significant $(P=.89)$. For asymmetric ossification, the only statistically significant difference was at the seventh decade with the rate favoring men $(P=.02)$.

Calcification rates did not reveal statistically significant difference among age groups for each sex.

When we evaluated the overall mineralization between 18 and 39 years of age, it was more common in men but with borderline statistical significance $(P=.05)$. After age 40 , it was more common in women but again with no statistically significant difference $(P=.4)$. Only for the group of patients who were older than 60 years of age was mineralization seen as statistically significantly higher in women $(P=.03)$. The same statistical significance was found for asymmetric mineralization, also favoring women $(P=.04)$.

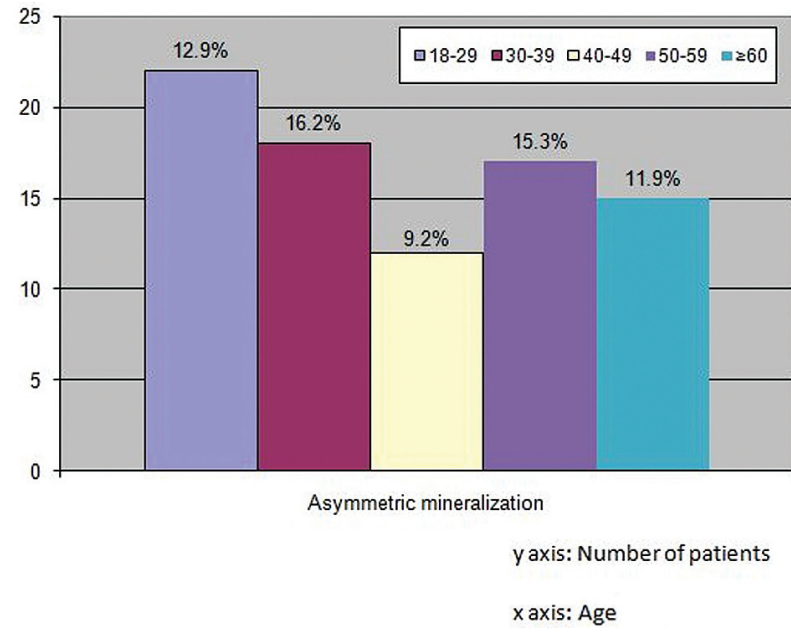

Fig 8. Age-specific rates of asymmetric arytenoid mineralization.

\section{Discussion}

The laryngeal cartilages are hyaline cartilages, with the exception of the epiglottis and vocal process of the arytenoid, which are fibroelastic cartilages. ${ }^{5,6}$ Hyaline cartilages undergo changes with time, with progressive enchondral ossification. ${ }^{7,8}$ Histopathologic studies demonstrated that the stage of the calcification and ossification is widely affected by age. ${ }^{9}$ The order of ossification is affected by the distribution of the mechanical forces applied to the laryngeal cartilages. ${ }^{8}$ Ossification begins first in the superior border of the lamina in the cricoid cartilage followed by the apex, body, and muscular process of the arytenoid cartilage, with the exception of the vocal process. ${ }^{10,11}$ Our aim was not to elucidate the pattern in which arytenoid ossification progresses, but we agree with the previously published observation that ossification initially occurs in the lateral one-third of the arytenoids peripherally and progresses to involve the center afterward. ${ }^{10,11}$ This is in concordance with the hypothesis that the order of ossification is affected by the distribution of mechanical forces applied to the laryngeal cartilages, ${ }^{8}$ because the lateral and posterior cricoarytenoid muscles attach to the lateral part (muscular process) of the arytenoid.

CT contributes greatly to the evaluation of the larynx and patients with laryngeal cancer. However because of the extreme variations in mineralization, CT has failed in the detection of minor cartilage invasion. MR imaging was at first deemed very promising but has been a disappointment because of false-positive imaging features that are attributed to inflammation mimicking neoplastic spread. ${ }^{3,12}$

The literature has demonstrated the nonossified (the mature cartilage), ossified (partial or complete), and sclerotic CT appearances of the cartilage. ${ }^{12,13}$ Ossified cartilage shows a high-attenuation outer and inner cortex with a central lowattenuation marrow. Sclerotic cartilage exhibits obliteration of that marrow with attenuated calcification, whereas the attenuation values of nonossified hyaline and fibroelastic cartilage are similar to those of soft tissue. We classified the mineralization process as calcification, sclerosis, and ossification.

In a prior study of 100 patients, a sclerotic arytenoid cartilage was observed in $16 \%$ compared with $19.4 \%$ in our study. ${ }^{4}$ Female and left side predominance was observed in this study as well as ours, but the reasons for these findings are unknown. 
Among the pathologic conditions causing arytenoid sclerosis, squamous cell carcinoma is perhaps the most important one. The process of neoplastic invasion of laryngeal cartilages involves 3 phases $^{12}$ : inflammatory changes within the cartilage adjacent to tumor inducing new bone formation before actual tumor invasion, osteolysis, and actual invasion by tumor cells. ${ }^{11}$ Increased osteoblastic activity and new bone formation, therefore, may become evident even before the tumor penetrates the perichondrium, which cannot be differentiated from tumor invasion by using MR imaging or CT. Becker et $\mathrm{al}^{12}$ found that the specificity of sclerosis for invasion by squamous cell cancer is lower in the thyroid cartilage (40\%) and higher in the cricoid (76\%) and arytenoid cartilages (79\%).

Several CT findings have been used to identify cartilage invasion: cartilage sclerosis, lysis, erosion, irregular contour, and cartilage blowout. ${ }^{12,13}$ Becker et al $^{12}$ reported that isolated arytenoid cartilage sclerosis as the sole criterion had a $68 \%$ sensitivity and $79 \%$ specificity for the prediction of invasion by squamous cell cancer. A comparative study of preoperative CT staging versus histopathologic staging in 38 patients showed $45 \%$ overstaging and $10 \%$ understaging of the total laryngectomy specimens. ${ }^{14}$ In 14 of the 17 erroneously upstaged tumors, arytenoid cartilage sclerosis with adjacent tumor on CT was the most common reason. ${ }^{14}$ The most common place of invasion in the arytenoid cartilage is at its base and at the level of the CAJs, where attachments of collagen bundles (Sharpney fibers) interrupt the perichondrium. ${ }^{11}$ Ossified cartilage is generally believed to be much more susceptible to tumor invasion than nonossified cartilage due to the presence of a tumor angiogenetic factor and acquired development of blood supply. ${ }^{12}$

The presence of asymmetric mineralization of the arytenoid cartilages in cases of laryngeal cancer may lead to erroneous characterization of the less mineralized arytenoid as being lytic. Thirteen percent of the subjects in this study showed asymmetric mineralization of the arytenoids.

In addition to laryngeal cancer, there are various uncommon conditions affecting the arytenoids as well as the CAJs. The CAJ is a diarthrotic joint with a joint capsule supported by a ligament and lined by a synovial membrane. ${ }^{15}$ The CAJ may harbor changes comparable with osteoarthritic changes seen other joints. ${ }^{16,17}$ Usually the affected CAJ undergoes ankylosis manifesting as immobility. ${ }^{18}$ There are many reports of involvement of the larynx and more specifically the CAJ by various systemic diseases; however, there are no references in those reports to radiologic correlates of such involvement. These disorders include rheumatoid arthritis, ${ }^{18}$ ankylosing spondylitis, ${ }^{19}$ juvenile chronic arthritis, ${ }^{20}$ autoimmune hepatitis, ${ }^{21}$ pemphigus, ${ }^{22}$ herpes zoster, ${ }^{23}$ tuberculosis, ${ }^{24}$ Wegener granulomatosis, ${ }^{24}$ Crohn disease,${ }^{25}$ syphilis, ${ }^{25}$ gout, ${ }^{26}$ sarcoidosis, ${ }^{27}$ and Sjogrens syndrome. ${ }^{28}$

Most of the etiologies mentioned above usually manifest without a mass. Vocal process granuloma usually presents as a mass at the posterior glottic mucosal surface and may present, particularly in the late stage, as sclerosis of adjacent arytenoid cartilage on $\mathrm{CT}^{29}$

Chondroma and chondrosarcoma are rare entities that may present with sclerotic cartilages, though most of these occur in the cricoids and arytenoid involvement is extremely rare..$^{30,31}$
There are some limitations of our study. Our study population mostly consisted of patients with trauma being evaluated in the emergency department. We reviewed the patients' charts to exclude a history of laryngeal cancer and other conditions that may potentially affect the laryngeal cartilages, though there may be instances in which some of these may have not been included in emergency department notes. The retrospective nature of the study may be considered a limitation, though all the CT studies were performed on the same scanner with similar protocols. Some sex and age groups have fewer participants (eg, men, 30-40 years of age), which may have affected the statistical calculations. We did not measure interreader or intrareader variability, though given the relative ease of identification of calcification on CT, we suspect that these have little, if any, effect on our results.

\section{Conclusions}

Asymmetric mineralization of the arytenoid cartilages was seen in $12.9 \%$ of our study population. The rate of unilateral arytenoid sclerosis was $4.6 \%$ in all subjects, $6.6 \%$ in women, and $3.5 \%$ in men. Unilateral sclerosis is much more frequently associated with the left arytenoid compared with the right. This association should be taken into account when evaluating CT scans of patients with laryngeal cancer for arytenoid cartilage invasion to avoid false-positive reads.

\section{References}

1. Yousem DM, Tufano RP. Laryngeal imaging. Magn Reson Imaging Clin N Am 2002;10:451-65

2. Farrag TY, Koch WM, Cummings CW, et al. Supracricoid laryngectomy outcomes: the Johns Hopkins experience. Laryngoscope 2007;117:129-32

3. Becker M, Zbaren P, Casselman JW, et al. Neoplastic invasion of laryngeal cartilage: reassessment of criteria for diagnosis at MR imaging. Radiology 2008;249:551-59

4. Schmalfuss IM, Mancuso AA, Tart RP. Arytenoid cartilage sclerosis: normal variations and clinical significance. AJNR Am J Neuroradiol 1998;19:719-22

5. Yeager VL, Lawson C, Archer CR. Ossification of the laryngeal cartilages as it relates to computed tomography. Invest Radiol 1982;17:11-19

6. Kahane JC, Kahn AR. India ink pinprick experiments on surface organization of cricoarytenoid joints. J Speech Hear Res 1986;29:544-48

7. Cohen SR, Cheung DT, Nimni ME, et al. Collagen in the developing larynx: preliminary study. Ann Otol Rhinol Laryngol 1992;101:328-32

8. Turk ML, Hogg DA. Age changes in the human caryngeal cartilages. Clin Anat $1993 ; 6: 154-62$

9. Casiano RR, Ruiz PJ, Goldstein W. Histopathologic changes in the aging human cricoarytenoid joint. Laryngoscope 1994;104(5 pt 1):533-38

10. Hatley W, Samuel E, Evison G. The pattern of ossification in the laryngeal cartilages: a radiological study. Br J Radiol 1965;38:585-91

11. Dedivitis RA, Abrahão M, Simões MJ, et al. Aging histological changes in the cartilages of the cricoarytenoid joint. Acta Cir Bras 2004;19:136-40

12. Becker M, Zbaren P, Delavelle J, et al. Neoplastic invasion of the laryngeal cartilage: reassessment of criteria for diagnosis at CT. Radiology 1997;203: $521-32$

13. Katilmiçs H, Oztürkcan S, Ozdemir I, et al. A clinico-pathological study of laryngeal and hypopharyngeal carcinoma: correlation of cord-arytenoid mobility with histopathologic involvement. Otolaryngol Head Neck Surg 2007; 136:291-95

14. Agada FO, Nix PA, Salvage D, et al. Computerised tomography vs. pathological staging of laryngeal cancer: a 6-year completed audit cycle. Int J Clin Pract 2004;58:714-16

15. Cerat J, Charlin B, Brazeau-Lamontagne L, et al. Assessment of the cricoarytenoid joint: high-resolution CT scan study with histo-anatomical correlation. J Otolaryngol 1988;17:65-67

16. Bullough $\mathrm{P}$, Goodfellow $\mathrm{J}$. The significance of the fine structure of articular cartilage. J Bone Joint Surg Br 1968;50:852-57

17. Tillmann B, Schunke M. Pathology of osteoarthritis. In: Hirohata K, Mizuno K, Matsubara T, eds. Trends in Research and Treatment of Joint Diseases. New York: Springer-Verlag; 1992:20-28

18. Chen JJ, Branstetter BF 4th, Myers EN. Cricoarytenoid rheumatoid arthritis: an important consideration in aggressive lesions of the larynx. AJNR Am J Neuroradiol 2005;26:970-72 
19. Helfgott SM, Treseler PA. Cricoarytenoid synovitis in ankylosing spondylitis. Arthritis Rheum 1990;33:604-05

20. Bertolani MF, Bergamini BM, Marotti F, et al. Cricoarytenoid arthritis as an early sign of juvenile chronic arthritis. Clin Exp Rheumatol 1997;15:115-16

21. Loukili NH, Pettorin C, Noel E, et al. Type 1 autoimmune hepatitis revealed by a dysphonia related to cricoarytenoid arthritis. QJM 2003;96:171-72

22. Vasiliou A, Nikolopoulos TP, Manolopoulos L, et al. Laryngeal pemphigus without skin manifestations and review of the literature. Eur Arch Otorhinolaryngol 2007;264:509-12

23. Morinaka S. Herpes zoster laryngitis with intractable hiccups. Auris Nasus Larynx 2009;36:606-08. Epub 2009 Mar 4

24. Loehrl TA, Smith TL. Inflammatory and granulomatous lesions of the larynx and pharynx. Am J Med 2001;111 (suppl 8A):113S-17S
25. Yang J, Maronian N, Reyes $\mathrm{V}$, et al. Laryngeal and other otolaryngologic manifestations of Crohn's disease. J Voice 2002;16:278-82

26. Montgomery WW. Cricoarytenoid arthritis. Laryngoscope 1963;73:801-36

27. Neel HB 3rd, McDonald TJ. Laryngeal sarcoidosis: report of 13 patients. Ann Otol Rhinol Laryngol 1982;91(4 pt 1):359-62

28. Seve P, Poupart M, Bui-Xuan C, et al. Cricoarytenoid arthritis in Sjogren's syndrome. Rheumatol Int 2005;25:301-02

29. Benjamin B, Roche J. Vocal granuloma, including sclerosis of the arytenoid cartilage: radiographic findings. Ann Otol Rhinol Laryngol 1993;102:756-60

30. Stiglbauer R, Steurer M, Schimmerl S, et al. MRI of cartilaginous tumours of the larynx. Clin Radiol 1992;46:23-27

31. Rizzo S, Strinati F, Longari F, et al. Chondrosarcoma of the larynx: presentation of a case and review of the literature. Tumori 2008;94:864-68 